\title{
Tratamiento de la boca seca. Nuevas tendencias
}

\section{Treatment of dry mouth. New trends}

\author{
Jané Salas E*, Estrugo Devesa A*, Ayuso Montero R**, López López J*
}

\begin{abstract}
RESUMEN
La disminución de saliva afecta la calidad de vida de los pacientes, que pueden mejorar cambiando ciertos hábitos de salud general como hidratación, uso de humidificadores ambientales, evitar tóxicos y conductas saludables, incluyendo visitas frecuentes al dentista. La prevención en los pacientes que van a ser irradiados, la modificación de los fármacos xerostomizantes en los casos posibles contribuyen al bienestar de estos pacientes. Los diferentes métodos de estimulación salivar tanto farmacológicos (ácidos, pilocarpina) como no farmacológicos (mecánicos, eléctricos, láser, acupuntura u oxígeno hiperbárico) y el uso de sustitutos salivales ayudan a mantener mejor hidratación bucal. En el futuro, el desarrollo de componentes orgánicos a partir de células madre será probablemente una posibilidad de tratamiento efectiva.
\end{abstract}

Palabras clave: Tratamiento de la boca seca, boca seca.

\section{SUMMARY}

Decreased saliva affects patients quality of life, that can improve changing certain health habits like hydration, usin groom humidifiers, avoid toxics and healthy behaviors, including frequent visits to the dentist. Prevention in patients who are going to be irradiated and modify xerostomizing drugs where possible contribute to the welfare of these patients. Different salivary stimulation methods, both pharmacological (acid, pilocarpine) and non-pharmacological (mechanical, electrical, laser, acupuncture or hyperbaric oxygen) and the use of salivary substitutes help maintain oral moisture better. In the future, the development of organic components from stem cells will probably be a valid line of treatment.

Key words: Dry mouth treatment, dry mouth.

Fecha de recepción: 15 de enero de 2014.

Aceptado para publicación: 19 de marzo de 2014.

* PhD, DDS, MD. Profesor de Medicina Oral. Facultad de Odontología. Universidad de Barcelona.

** PhD, DDS. Profesor de Prótesis Dental. Facultad de Odontología. Universidad de Barcelona.

Jané Salas E, Estrugo Devesa A, Ayuso Montero R, López López J. Tratamiento de la boca seca. Nuevas tendencias. Av. Odontoestomatol 2014; 30 (3): 135-138.

\section{INTRODUCCIÓN}

La boca seca es un problema de magnitud creciente y que conlleva una serie de alteraciones que afectan, en último término, la calidad de vida de los pacientes; es por ello que su tratamiento supone un reto para el profesional (1). La orientación terapéutica así como su pronóstico depende de la etiología que origina el problema. Los tratamientos deben enfocarse diferenciando las causas transitorias de las permanentes, así como de la cantidad de parénquima glandular funcional remanente del paciente. A lo 
laro de este trabajo iremos describiendo los aspectos más novedosos de su tratamiento.

\section{MEDIDAS GENERALES}

Ante cualquier paciente que acuda refiriendo sensación de boca seca, aparte de realizar una historia clínica muy detallada, buscando fundamentalmente todos aquellos antecedentes de tipo clínico y farmacológicos (de gran importancia) que puedan orientar la etiología del proceso (2), y de realizar las pruebas complementarias para diagnosticar al paciente de una alteración funcional u orgánica, se deberán iniciar una serie de medidas, que en un inicio serán iguales para todos los pacientes (Tabla 1).

\section{MODIFICACIÓN DE FÁRMACOS}

La farmacología, actualmente es uno de los pilares terapéuticos más importantes asociado a la longevidad del ser humano, como es lógico pensar todo fármaco posee acciones terapéuticas deseadas y unos efectos secundarios, que en nuestro ámbito de actuación provocan fundamentalmente sequedad de boca, siendo los fármacos más comúnmente asociados los hipotensores y los que actúan a nivel del

TABLA 1.- MEDIDAS BÁSICAS DE CONTROL ANTE UN PACIENTE CON XEROSTOMÍA

- Hidratación correcta: Agua, bebidas no carbonatadas ni azucaradas.

- Ambientes con saturación elevada de agua: Evitar aires acondicionados y calefacciones. Utilización de humidificadores ambientales.

- Evitar los hábitos tóxicos que agravan el proceso: Alcohol, tabaco inhalado o masticado, café, té o bebidas con extractos de cola, etc.

- Conductas saludables: Dietas poco especiadas, ejercicio regular.

- Visitas periódicas al dentista para diagnosticar lesiones del esmalte incipientes y de la mucosa. Realización de profilaxis frecuentes. sistema nervioso central y que poseen cierto efecto anticolinérgico.

Desde el punto de vista teórico, y si fuese posible, deberían modificarse estas medicaciones para intentar revertir el efecto xerostomizante que provocan, pero ello no siempre es fácil y debemos asumir que el efecto principal supera el efecto secundario y entonces, aparte de las medidas generales, deberemos iniciar tratamientos sustitutorios ya que muchos fármacos sialogogos no son compatibles con las medicaciones antes mencionadas.

\section{PREVENCIÓN}

Los tratamientos de radioterapia en pacientes con cáncer de cabeza y cuello provocan, dependiendo de la dosis, una destrucción de las glándulas salivales, sobre todo cuando la dosis total recibida supera los 60 Gy, clínicamente se traduce con la aparición de una xerostomía progresiva que desemboca en una asialia de tipo irreversible. En estos pacientes, aparte de intentar realizar todos los tratamientos odontológicos precisos previos al inicio del procedimiento, pueden mitigarse los efectos con las nuevas técnicas de radioterapia, nos referimos a la utilización de Radiación de Intensidad Modulada, que irradia en menor medida las zonas glandulares.

En casos que los campos a irradiar sean muy extensos, se ha probado con éxito el autotrasplante de glándula submaxilar a la zona submental, retirándola de la zona de máxima irradiación y preservándola (3).

\section{ESTIMULACIÓN GLANDULAR NO FARMACOLÓGICA}

En los pacientes con xerostomía y alguna reserva salival, la utilización de elementos de tipo mecánico, como huesos de aceituna, cereza, etc. provoca un efecto estimulante local que puede dar una sensación de mejora al paciente. La utilización del láser de baja potencia (4) en glándulas salivales se ha empleado en pacientes con xerostomía y según la literatura reciente se puede obtener una estimulación de las glándulas salivales, comparándolo con la es- 
timulación con ácido cítrico. Además, se ha propuesto que su utilización puede tener un efecto regenerativo sobre las glándulas.

La electroestimulación se basa en que la estimulación eléctrica del nervio lingual produce una contracción máxima de los ácinos glandulares aumentando el flujo salival en pacientes con síndrome de Sjögren. Los primeros estimuladores de gran tamaño se utilizaban en forma de electrodos que estimulaban directamente la lengua, posteriormente se utilizaron aparatos removibles de acrílico que contienen una batería con un estimulador lingual, y últimamente se ha propuesto la utilización de implantes con estimuladores integrados en pacientes con ausencia de molares inferiores. Se han descrito buenos resultados con la utilización de estos dispositivos (5). La acupuntura transcutánea se ha utilizado en pacientes irradiados, comparándolo con la utilización de pilocarpina, obteniendo mejores resultados y sin los efectos secundarios del fármaco. Dado el efecto angiogénico y cicatrizante que se le ha supuesto a la utilización de oxígeno hiperbárico (6) se ha intentado utilizar en estos pacientes, sobre todo en t irradiados, logrando, según diferentes estudios, una mejoría notable como mínimo en el $50 \%$ de los pacientes estudiados con aumento significativo del flujo salival.

\section{ESTIMULACIÓN GLANDULAR FARMACOLÓGICA}

Clásicamente la utilización de elementos ácidos se ha utilizado como estimulante salival, y de ellos el más conocido es el ácido cítrico, bien en forma de comprimidos o en solución, que utilizado al $2 \%$ tiene un efecto estimulante muy importante sobre la secreción salival estimulada, aunque es conocido que no modifica la secreción basal. Dentro de este grupo de estimulantes tópicos directos también se ha utilizado el ácido málico, que posee una utilidad semejante al anterior, y que ha demostrado su efectividad en pacientes ancianos polimedicados. Dentro de este grupo no podemos dejar de citar al fármaco con acción sialogoga por excelencia que es la pilocarpina, que utilizada en gotas o por vía sistémica provoca una estimulación directa sobre las glándulas salivales por su efecto parasimpaticomimético, se utiliza a dosis de $5 \mathrm{mg}$, tres veces al día, y por un periodo que no debe superar los 2-3 meses.

La aparición de sudoración, escalofríos, rinitis, síndrome gripal y otros derivados de sus efecto sistémico farmacológico se consideran habituales y, dependiendo de la intensidad de los mismos, pueden motivar el abandono del tratamiento. Cevimeline es un fármaco parasimpaticomimético agonista muscarínico, no comercializado en nuestro país, con efecto superponible a la pilocarpina y que se utiliza en pacientes con síndrome de Sjögren; las limitaciones son las mismas y los efectos secundarios, menores. También se ha utilizado la hidroxicloroquina, bromhexina y betanecol con efectos muy cuestionables y sin mejora aparente respecto a la utilización de otros fármacos comentados anteriormente (7-9).

\section{SUSTITUTOS SALIVALES}

En los casos que las medidas anteriores no hayan tenido la eficacia esperada o para tratamientos concomitantes con los anteriores o ante la ausencia total de parénquima glandular residual remanente, el único medio que tenemos a nuestro alcance para mejorar la calidad de vida de estos pacientes es la utilización de productos que remeden a la saliva, tenemos desde los sistemas con complejos lactoperoxidasa, a toda una nueva línea de productos que contienen aceite de oliva, betaína y xilitol, que persiguen por un lado lubricar la cavidad oral, dotándola de una capacidad tampón y así evitar gran parte de los efectos secundarios que produce la ausencia de saliva, como son la aparición de caries o de infecciones fúngicas, y, por otro lado, mejorar la capacidad deglutoria y fonatoria de los pacientes afectados (10). De entre los muchos sustitutos salivales, tan solo dos, según refiere López-López et al (2), presentan estudios bien estructurados, son los del grupo OralBalance ${ }^{\circledR}$ y el Xerostom ${ }^{\circledR}$.

\section{EL FUTURO}

La ingeniería genética ha logrado en los últimos años abrir un campo de esperanza en la generación de componentes orgánicos a partir de células madre pluripotenciales, este progreso ha dado lugar a un nuevo 
concepto, el de Medicina Regenerativa que, sin duda, es no ya el futuro sino, en muchos campos, el presente de la investigación biomédica. Podemos decir que se han desarrollado ya en el laboratorio elementos funcionales del riñón, las nefronas y, en estudios en ratones, el crecimiento de glándulas compatibles tanto en histología como en función a las salivales que en un futuro, esperemos que no muy lejano, podrá permitir que, en aquellos pacientes que por problemas terapéuticos o primarios como las enfermedades de estirpe inmunológica, puedan beneficiarse de este campo médico en constante expansión (11).

\section{BIBLIOGRAFÍA}

1. Tim Dijkema T,Raaijmakers C, Braam P,Roesink JM, Monninkhof EM, Terhaard CHJ. Xerostomia: A day and night difference. Radiotherapy and Oncology 2012;104:219-23.

2. López López J, Jané Salas E, Chimenos Küstner E. Pronóstico y tratamiento de la boca seca. Revisión sistemática. Medicina Clínica (Barcelona). 2013; 7753:269-78.

3. Zhang Yen, Guo Chuan-Bin, Zhang Lei, Wang Yang, et al. Prevention of radiation-induced xerostomia by submandibular gland transfer. Head E Neck 2012; 34:937-42.

4. Loncar B, Stipetic MM, Baricevic M, Risovic D. The Effect of Low-Level Laser Therapy on Salivary Glands in Patients with Xerostomia 2011;29:171-5.

5. Fedele S, Wolff A, Strietzel F, Martín-Granizo López $\mathrm{R}$, Stephen R, Porter and Yrjöt Konttinen. Neuroelectrostimulation in Treatment of Hyposalivation and Xerostomia in Sjögren's Syndrome: A Salivary Pacemaker. J Rheumatol. 2008;35;1489-94.

6. Cankar K, Finderle Z, Jan J. The Effect of Hyperbaric Oxygenation on Postradiation Xerostomia and Saliva in Patients with Head and Neck Tumours. Caries Res 2011;45:136-41.
7. Silvestre FJ, Mínguez MP, Suñe-Negre JM. Clinical evaluation of a new artificial saliva in spray form for patients with dry mouth. Med Oral Patol Oral Cir Bucal 2009;1:e8-11.

8. Femiano F, Rullo R, di Spirito F, Lanza A, Festa VM, Cirillo N. A comparison of salivary substitutes versus a natural sialogogue (citric acid) in patients complaining of dry mouth as an adverse drug reaction: A clinical, randomized controlled study. Oral Surg Oral Med Oral Pathol Oral Radiol Endod 2011;112:e15-20.

9. Gómez-Moreno G, Aguilar-Salvatierra A, Guardia J, Uribe-Marioni A, Cabrera-Ayala M, Arcesio Delgado-Ruiz R, et al. The efficacy of a topical sialogogue spray containing $1 \%$ malic acid in patients with antidepressant-induced oral dry mouth: adouble-blind, randomized clinical trial. Depression and Anxiety 2013;30: 137-42.

10. Shahdad SA, Taylor C, Barclay SC, Steen IN, Preshaw PM. A double-blind, crossover study of Biotene Oralbalance and BioXtra systems as salivary substitutes in patients with post-radiotherapy xerostomia. Eur J Cancer Care (Engl) 2005;14: 319-26.

11. Pringle S, Van Os R, Coppes RP. Concise Review: Adult Salivary Gland Stem Cells and a Potential Therapy for Xerostomia. Stem Cells 2013;31: 613-9.

\section{CORRESPONDENCIA}

Dr. José López López

Campus Universitario de Bellvitge

Departamento de Odontoestomatología

Facultad de Odontología

Pabellón de Gobierno

Feixa Llarga, s/n

08907 L'Hospitalet de Llobregat, Barcelona

Correo electrónico: 18575jll@gmail.com 